\title{
A Description of Clinical Decision Support Framework Prediction in Heterogeneous Data Sources
}

\author{
P.Punithavalli, P.Sathish Kumar, S.Sivaraman
}

\begin{abstract}
To keep pace with the updates in obliging scientific discipline, thriving recuperating knowledge is being assembled incessantly. Regardless, inferable from the not too appalling gathering of its categories and sources, therapeutic knowledge has over up being significantly hugger-mugger in numerous specialist's work environments that it currently wants Clinical call Support (CDS) system for its affiliation. To reasonably utilize the party flourishing knowledge, we tend to propose a CDS structure which will distort mixed thriving knowledge from totally different sources, for example, take a goose at workplace check works out as planned, important info of patients and action records into a joined depiction of options everything thought-about. Victimization the electronic roaring healing knowledge therefore created, multi-name delineation was accustomed endorse a layout of afflictions and so facilitate consultants in diagnosis or treating their patients' therapeutic problems a lot of competently. Once the ace sees the contamination of a patient, the running with organize is to contemplate the conceivable complexities of that disarray, which may impel a lot of infections.
\end{abstract}

Keywords - Clinical decision support system, heterogeneous

\section{INTRODUCTION}

Health knowledge from various medical sources (CyberPhysical Systems, the web of Things) is collected endlessly; facilitating the expansion of care business. It is wide accepted that health data tools and machine learning techniques is exploited with success to assist doctors in identification and treating their patients additional expeditiously. Victimization their expertise and information, the physicians classify patients and diagnose their diseases, however in doing this, it's probable that they commit some mistakes, significantly after they lack adequate expertise or once their school of judgment is poor. In such things, Clinical call Support (CDS) systems, as well as systems that give designation, customized medical mensuration, treatment and relevant information, would be useful to the physicians by approach of providing them with specific information, patients' data and intelligent applications, which might improve the potency of their decision-making processes.

Revised Manuscript Received on August 14, 2019.

P.Punithavalli, PG Scholar, Computer Science and Engineering, Vivekananda College of Engineering for Women, Tiruchengode, India. (Email: punithakalai941@gmail.com)

P.SathishKumar, Assistant Professor, Computer Science and Engineering, Vivekananda College of Engineering for Women, Tiruchengode, India. (E-mail: p.sathishkts@gmail.com)

S.Sivaraman, Assistant Professor, Computer Science and Engineering, Vivekananda College of Engineering for Women, Tiruchengode, India. (E-mail: sivaramanbtech@gmail.com) data sources, Diagnosis recommender systems.

CDS systems specialize in extracting characteristics of patients, supported that they classify patients and supply corresponding clinical suggestions to the physicians. Patients' medical data is extracted from this personal medical knowledge, like the physiological knowledge, electronic health records (EHRs), 3D images, radiology pictures, genomic sequencing, and clinical and request knowledge. Through CDS applications, the physicians will avoid the mistakes that are probably to arise from medical negligence and therefore improve the standard of their medical service. Within the medical field, the demand for high-quality clinical support systems has been steady on the rise. In medical scenes, the specifies of rating standards and therefore the context quality of medical field are the challenges of clinical call web.

\section{RELATED WORK}

The health medical knowledge of such an oversized range of patients would clearly be of peta or letter bytes, and this refers to the quantity of massive knowledge. The patient's info is updated as before long as he/she visits the hospital once more and this represents the rate of health medical knowledge. Medical knowledge consists of structured, semistructured and unstructured knowledge. Moreover, the health medical knowledge is of various categories: electronic health medical records written by physicians; knowledge from period monitors; pictures collected by CAT (CT); nuclear resonance pictures (MRI); viscus angiographs etc. every patient's medical records from skilled physicians and medical instruments replicate his/her real vigor, that represent the truthfulness of health medical knowledge. The cooperativeness of the collected health knowledge for transformation into helpful and meaty information, that represents the worth of the information. Therefore, health medical knowledge may be a reasonably "Big data" to some extent.

Nowadays, the data-intensive applications need an oversized range of economical models. Various random strategies were exploited by completely different researchers for care parameter analysis. Moreover, physicians contemplate the similarity between the health parameters of a patient for correct diagnosing call. Analysis of massive knowledge is applied in care to spot clusters of patients and teams of diseases, that square measure accustomed estimate

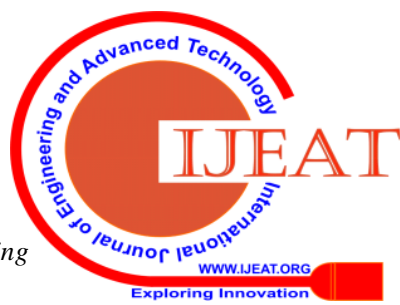




\section{A DESCRIPTION OF CLINICAL DECISION SUPPORT FRAMEWORK PREDICTION IN HETEROGENEOUS DATA SOURCES}

future health condition with the assistance of various machine learning techniques. The CDS system will utilize acceptable computing technology to boost the potency of decision-making. The big knowledge of health has been recognized as an excellent chance for rising CDS systems.

\section{EXISTING SYSTEM}

Existing conception deals with providing backend by victimization MYSQL that contains heap of drawbacks i.e., knowledge limitation is that time interval is high once is large and once data is lost we tend to cannot recover therefore tends to proposing conception by victimization Hadoop framework.

\subsection{DRAWBACKS}

- Limitation of knowledge.

- To get results with take longer and maintenance price is extremely high.

\section{PROPOSED SYSTEM}

Proposed thought deals with providing information by exploitation Hadoop tool we will analyze no limitation of information and straight forward add range of machines to the cluster and get results with less time, high turnout and maintenance price is incredibly less and that we area unit exploitation partitions and bucketing techniques in Hadoop.

\subsection{SYSTEM ARCHITECTURE}

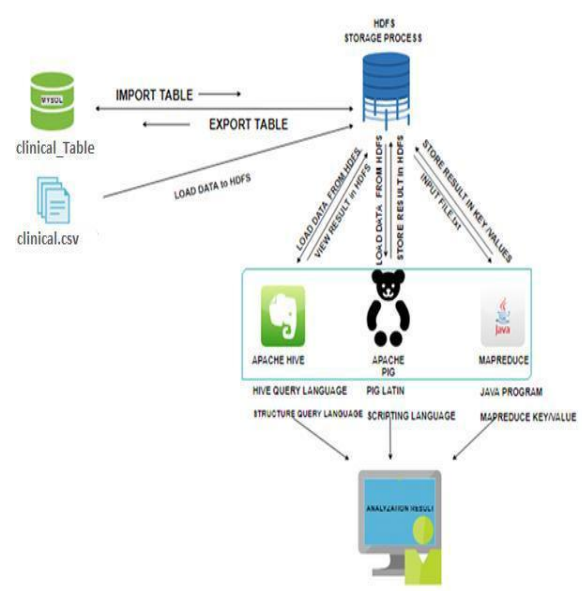

Figure 4.1 System Architecture

The architecture is large amount of clinical data set to snoop command designing tools is will be transferring bulk of data is stored in Hadoop system. Map-reduce is divided into two task one is map task and reduce task. Map task is the processes the data and creates several small chunks of data. The Reducer task is to process the data that comes from the data mart. Pig provides many built-in operators to support data operations like grouping, filters, ordering, can be stored in data mart as result.

\subsection{ADVANTAGES}

- No data loss problem

- Efficient data processing.

\section{MODULES \& RESULTS}

\subsection{PREPROCESSING CLINICAL DATABASE}

In this module, analyzing the info with totally different varieties of fields in Microsoft stand out then it reborn into comma delimited format that is claimed to be csv (comma separator value) file and captive to MYSQL backup through information.

\subsection{PREPROCESSING}

Here by obtaining historical information to convert those historical instruction execution information from (.xlsx) format to (.csv) format and by taking backup of all this information in MYSQL info to avoid loss of knowledge.

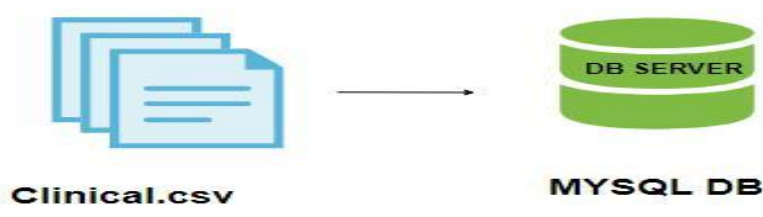

Figure 5.2 Preprocessing

\subsection{STORAGE}

In this module we tend to have gotten all those backup knowledge that I have keep in MYSQL and commercialism all that knowledge by use of sqoop commands to HDFS (Hadoop Distributed File System).now all the info are keep in HDFS where it's able to get processed by use of hive.

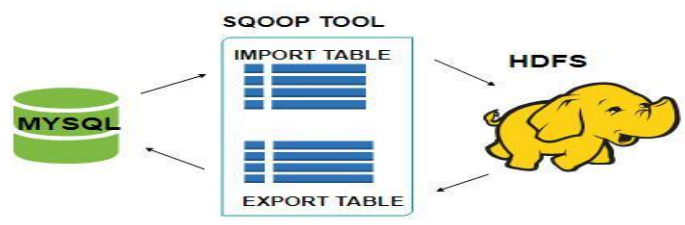

Figure 5.3 Storage

\subsection{ANALYSE QUERY}

In this module I tend to have gotten all this information from HDFS to HIVE by use of snoop import command. Were hived is prepared to research. Here in HIVE will method solely structured information to research. By extracting solely the meaty information and neglecting unclenched information we will analyze the information in more practical manner by use of hive.

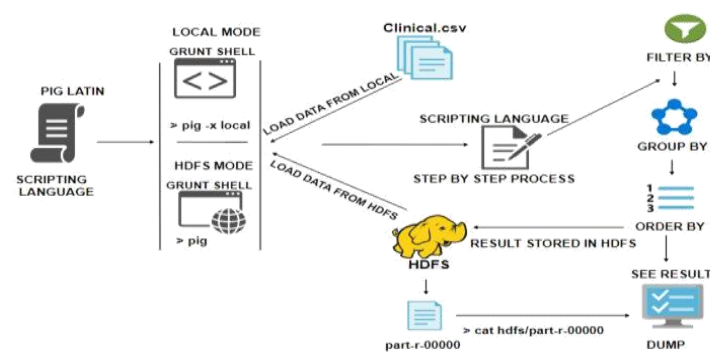

Figure 5.4 Analyse of query 


\subsection{ANALYSIS LATIN SCRIPT (PIG)}

To analyze Clinical mistreatment Pig, programmers have to be compelled to write scripts mistreatment Pig Latin language and execute them in interactive mode mistreatment the Grunt shell. Of these scripts area unit internally regenerate to Map and cut back tasks. After invoking the Grunt shell, you'll be able to run your Pig scripts' within the shell. Except LOAD and STORE, whereas performing arts all different operations, Pig Latin statements take a relation as input and turn out another relation as output. As shortly as you enter a Load statement within the Grunt shell, its linguistics checking is going to be applied. To check the contents of the schema, you would like to use the Dump operator. Solely once performing arts the dump operation, the MapReduce job for loading the info into the filing system are going to be applied. Pig provides several in built operators to support knowledge operations like grouping, filters, ordering, etc.

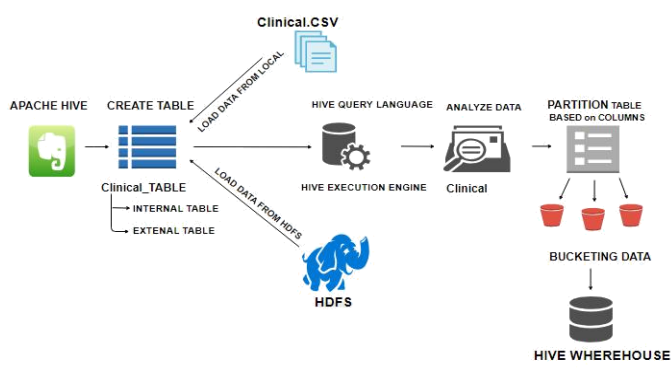

Figure 5.5 Analysis Latin Script

\subsection{PROCESSING (MAPREDUCE)}

MapReduce may be framework exploitation that we will write applications to method Brobdingnagian amounts of Clinical, in parallel, on massive clusters of goods hardware in a very reliable manner. MapReduce may be a process technique, and a program model for distributed computing supported java. The MapReduce rule contains 2 necessary tasks, particularly Map and cut back. MapReduce program executes in 3 stages, particularly map stage, shuffle stage, and cut backstage. The map or mapper's job is to method the input file. Usually the input file is within the type of file or directory and is keep within the Hadoop filing system (HDFS). The input data is passed to the clerk performs line by line. The clerk processes the info and creates many tiny chunks of knowledge. This stage is that the combination of the Shuffle stage and therefore the cut backstage. The Reducer's job is to method the info that comes from the clerk. When process, it produces a replacement set of output, which can be keep within the HDFS.

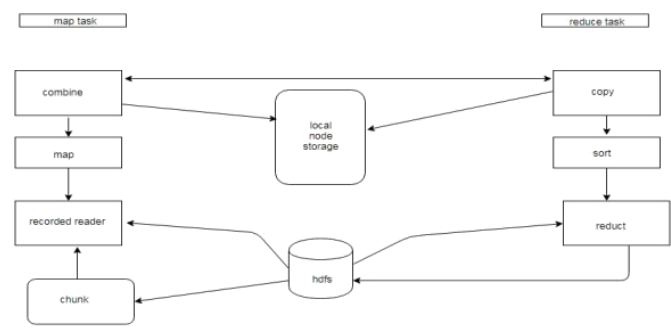

Figure 5.6 Processing

\section{CONCLUSION}

In this paper, I bestowed a study on Clinical is facilitated to doctors by obtaining plan what treatment he has given thereto explicit patient. To analysis the Clinical knowledge in Hadoop scheme. Hadoop scheme is hive, pig, Mapreduce. In future the spark a hundred times quicker than Hadoop, it's simply analysis quicker.

\section{FUTURE ENHANCEMENT}

Apache Spark is associate degree open supply process engine engineered around speed, case of use, and analytics. If you've got giant amounts of information that needs low latency process that a typical Map scale back program cannot offer, Spark is that the various. Spark provides inmemory cluster computing for lightning quick speed and supports Java, Scala, and Python arthropod genus for simple development.

\section{REFERENCES}

1. Hermann M, Pentek T, Otto B. Design Principles for Industrie 4.0 Scenarios, pp. 3928-3937, 2016.

2. Thuemmler C, Bai C. Health 4.0: How Virtualization and Big Data are Revolutionizing Healthcare, Springer International Publishing, 2017.

3. Cao P, Xian Medical University. "The Impact of Big Data on the Development of Chinese Health Service Industry", Journal of Electronic Test, 2014.

4. M. Akay, D. I. Fotiadis, K. S. Nikita and R. W. Williams, "Guest Editorial: Biomedical Informatics in Clinical Environments," Biomedical and Health Informatics, IEEE Journal of, vol. 19, no. 1, pp. 149-150, 2015.

5. Black A D, Car J, Pagliari C, et al. "The impact of eHealth on the quality and safety of health care: a systematic overview", PLoS Medicine, vol. 8, no.1, 2011.

6. Horsky J, Schiff G D, Johnston D, et al. "Methodological Review: Interface design principles for usable decision support: A targeted review of best practices for clinical prescribing interventions", Journal of Biomedical Informatics, vol. 45, no. 6, pp. 1202-1216, 2012.

7. Valdez A C, Ziefle M, Verbert $\mathrm{K}$, et al. "Recommender Systems for Health Informatics: State-of-the-Art and Future Perspectives", Machine Learning for Health Informatics, Springer International Publishing, 2016.

8. $\mathrm{Yu} \mathrm{W} \mathrm{D,} \mathrm{Pratiksha} \mathrm{C,} \mathrm{Swati} \mathrm{S,} \mathrm{et} \mathrm{al.} \mathrm{"A} \mathrm{Modeling} \mathrm{Approach}$ to Big Data Based Recommendation Engine in Modern Health Care Environment", Computer Software and Applications Conference, IEEE Computer Society, pp.75-86, 2015.

9. Bhatti U A, Huang M, Zhang Y, et al. Research on the Smartphone Based eHealth Systems for Strengthening Healthcare Organization[M]// Smart Health. 2017.

10. Vancampfort D, Mugisha J, Hallgren M, et al. "The prevalence of diabetes mellitus type 2 in people with alcohol use disorders: a systematic review and large scale metaanalysis”, Psychiatry Research, vol. 246, pp. 394-400, 2016. 